International Journal of Pure and Applied Mathematics

Volume 110 No. 1 2016, 165-182

ISSN: 1311-8080 (printed version); ISSN: 1314-3395 (on-line version)

url: http://www.ijpam.eu

doi: 10.12732/ijpam.v110i1.16

ijpam.eu

\title{
N-SYMMETRY OF ITô STOCHASTIC DIFFERENTIAL EQUATION DRIVEN BY POISSON PROCESS
}

\author{
Aminu M. Nass ${ }^{1}$, E. Fredericks ${ }^{2}$ \\ ${ }^{1,2}$ Department of Mathematics and Applied Mathematics \\ University of Cape Town \\ Private Bag X1, Rondebosch 7701, SOUTH AFRICA
}

\begin{abstract}
Lie point symmetry transformation of the class of Itô stochastic differential equation driven by Poisson Processes was successfully carried out. We consider symmetries involving not only spatial and time variables $(t, x)$, but also the Poisson process term $N(t)$. The result was achieved by following the invariance methodology of Lie point transformation and the use of Itô formula for Poisson stochastic differential equation without enforcing any conditions to the momenta of the stochastic process.
\end{abstract}

AMS Subject Classification: 60H10, 76M60

Key Words: Lie symmetries; Poisson process; stochastic differential equation; Itô formula.

\section{Introduction}

In [4] G. Gaeta extend his earlier work [6] to introduced W-symmetries by considering symmetries that involve both the spatial $x$, temporal variables $t$ and the vector Wiener process $w(t)$. However, G. Gaeta [4] enforced conditions that transformed Wiener process to be consistent with the original process in terms of its momenta, i.e., the instantaneous mean and variance of the transformed process are forced to be exactly the instantaneous mean and variance of the original Wiener process.

$\begin{array}{lr}\text { Received: } & \text { April 28, } 2016 \\ \text { Revised: } & \text { August 30, } 2016 \\ \text { Published: } & \text { October 21, } 2016\end{array}$

(c) 2016 Academic Publications, Ltd.

url: www.acadpubl.eu 
In this paper we extend [4] to the class of Itô stochastic differential equations driven by Poisson process

$$
d X(t, N)=f(t, X(t, N)) d t+J(t, X(t, N)) d N(t)
$$

Where $f(t, x)$ and $J(t, x)$ are the $n \times 1$ dimensional drift vector coefficient and Poisson diffusion coefficient respectively. Here $d N(t)$ is the infinitesimal increment of the Poisson process. We assume that the coefficient functions satisfy the Ikeda Watanabe conditions for the existence and uniqueness of the solution of $(1)[1,7]$.

Lie symmetry of (1) were discussed by extending [5] to consider infinitesimal generator that include the group transformations of Poisson process $N(t)$ i.e., we now consider infinitesimal involving not only the spatial and time variables $(t, x)$, but also the Poisson process variable $\mathrm{N}(\mathrm{t})$ i.e.,

$$
H=\tau(t, x, N) \frac{\partial}{\partial t}+\xi(t, x, N) \frac{\partial}{\partial x}+\phi(t, x, N) \frac{\partial}{\partial N} .
$$

The determining equations for Itô stochastic differential equation driven by Poisson processes (1) are successfully derived in an Itô calculus context, and they are found to be deterministic even though they represent a stochastic process.

The primary tools for finding admitted Lie point symmetry transformations for stochastic differential equation (SDEs) are Itô formula and the random change of time.

Theorem 1 (Itô Lemma for Poisson diffusion processm see [1, 2]). Let $Y(t)=F(t, X(t))$, such that the function $F(t, X(t))$ is once continuously differentiable with respect to $x$ and $t$. Let the $X(t)$ process satisfy the Itô stochastic differential equation with finite jump of the form,

$$
d X(t)=f(t, X(t)) d t+J(t, X(t)) d N(t)
$$

$X(0)=x_{0}$ where $f(t, X(t))$ and $J(t, X(t))$ are bounded and integrable respectively. Then

$d F(t, X(t))=\left(\frac{\partial F}{\partial t}+f \frac{\partial F}{\partial x}\right) d t+(F(t, X(t)+J(t, X(t)))-F(t, X(t))) d N(t)$.

Einstein summation convention is assumed through out this paper, for the matter of convenient let introduce operators

$$
\Gamma_{(F)}=\frac{\partial F}{\partial t}+f \frac{\partial F}{\partial x}
$$


and

$$
\Gamma_{(F)}^{*}=F(t, X(t)+J(t, X(t)))-F(t, X(t))
$$

and rewrite

$$
d F(t, X(t))=\left(\frac{\partial F}{\partial t}+f \frac{\partial F}{\partial x}\right) d t+(F(t, X(t)+J(t, X(t)))-F(t, X(t))) d N(t)
$$

as;

$$
d F(t, X(t, N))=\Gamma_{(F)} d t+\Gamma_{(F)}^{*} d N(t) .
$$

Finally, using Itô multiplication properties of stochastic differential equation driven by Poisson diffusion process $[1,2]$ and application of infinitesimal transformations the determining equations for the Poisson process stochastic differential equation (1) are derived. Precisely the following result was obtained.

Theorem 2. The Itô stochastic differential equation driven by Poisson processes

$$
d X(t)=f(t, X(t, N)) d t+J(t, X(t, N)) d N(t)
$$

where the coefficient functions $f(t, X(t))$ and $J(t, X(t))$ are $n \times 1$ dimensional drift vector coefficient and Poisson diffusion coefficients, with infinitesimal generator

$$
H=\tau(t, x, N) \frac{\partial}{\partial t}+\xi(t, x, N) \frac{\partial}{\partial x}+\phi(t, x, N) \frac{\partial}{\partial N} .
$$

has admitted the following determining equations;

$$
\begin{gathered}
(f \Gamma(\tau)+\lambda J(\Gamma(\tau)-\Gamma(\phi))+H(f)-\Gamma(\xi))(t, X(t, N))=0, \\
\left(J \Gamma^{*}(\phi)+H(J)-\Gamma^{*}(\xi)\right)(t, X(t, N))=0
\end{gathered}
$$

and

$$
\begin{gathered}
\Gamma(\phi)+\lambda \Gamma^{*}(\phi)=\lambda \Gamma(\tau) \\
\Gamma^{*}(\phi)=\frac{\Gamma(\tau)}{2}
\end{gathered}
$$

with additional conditions,

$$
\Gamma^{*}(\tau)=0 \quad \text { and } \quad \Gamma(\tau)=c_{1} .
$$

Where the operators $\Gamma(t, X(t, N))$ and $\Gamma^{*}(t, X(t, N))$ are defined as in (5) and $(6)$, and $\lambda>0$ is called the jump intensity. And the infinitesimals $\tau(t, x, N)$, $\xi(t, x, N)$ and $\phi(t, x, N)$ are called the admitted symmetries of (1) if and only if they satisfied the determining equations (11-15). 


\section{Lie Group Transformations}

Consider a one parameter group of transformation with time index $t$, the spatial variable $x$ and Poisson process variable $N$ respectively,

$$
\bar{t}=\theta_{1}(t, x, N, \epsilon) \quad \bar{x}=\theta_{2}(t, x, N, \epsilon) \quad \bar{N}=\theta_{3}(t, x, N, \epsilon)
$$

with the infinitesimals

$$
\frac{\partial \theta_{1}}{\partial \epsilon}=\tau\left(\theta_{1}, \theta_{2}, \theta_{3}\right), \quad \frac{\partial \theta_{2}}{\partial \epsilon}=\xi\left(\theta_{1}, \theta_{2}, \theta_{3}\right), \quad \frac{\partial \theta_{3}}{\partial \epsilon}=\phi\left(\theta_{1}, \theta_{2}, \theta_{3}\right)
$$

satisfying the following initial conditions at $\epsilon=0$

$$
\left.\bar{t}\right|_{\epsilon=0}=\left.t \quad \bar{X}\right|_{\epsilon=0}=\left.X \quad \bar{N}\right|_{\epsilon=0}=N .
$$

Where $\epsilon$ is the parameter of the group, hence the corresponding generator of the Lie algebra is of the form of

$$
H=\tau(t, x, N) \frac{\partial}{\partial t}+\xi(t, x, N) \frac{\partial}{\partial x}+\phi(t, x, N) \frac{\partial}{\partial N} .
$$

The group transformations can be expressed in term of of the symmetry operator (17) as

$$
\begin{aligned}
& \bar{t}=e^{\epsilon H}(t) \\
& \bar{x}=e^{\epsilon H}(x)
\end{aligned}
$$

and

$$
\bar{N}=e^{\epsilon H}(N)
$$

The Itô stochastic differential equation with Poisson related to the group of transformations are

$$
\begin{gathered}
d \bar{t}=\Gamma e^{\epsilon H}(t) d t+\Gamma^{*} e^{\epsilon H}(t) d N(t), \\
d \bar{N}=\Gamma e^{\epsilon H}(N) d t+\Gamma^{*} e^{\epsilon H}(N) d N(t),
\end{gathered}
$$

and

$$
d \bar{X}=\Gamma e^{\epsilon H}(x) d t+\Gamma^{*} e^{\epsilon H}(x) d N(t) .
$$

Using the Itô formula (8), we can also write the temporal, spatial and Poisson infinitesimals as Itô process respectively as,

$$
d \tau=\Gamma(\tau) d t+\Gamma^{*}(\tau) d N(t)
$$




$$
d \xi=\Gamma(\xi) d t+\Gamma^{*}(\xi) d N(t)
$$

and

$$
d \phi=\Gamma(\phi) d t+\Gamma^{*}(\phi) d N(t) .
$$

Where the operators $\Gamma(t, x)$ and $\Gamma^{*}(t, x)$ have been defined in (5) and (6) respectively, these operators are in fact instantaneous and standard deviation of the temporal, spatial and Poisson infinitesimals $\tau, \xi$ and $\phi$ respectively.

\subsection{Poisson Invariance Properties}

Before deriving the determining equations we apply the invariant to the moments of the Poisson processes to make sure it remain invariance under the group transformations, viz the instantaneous mean and variance of the Poisson process which are:

$$
\begin{gathered}
E_{Q}[d N(t)]=\lambda d t \\
E_{Q}[d N(t) d N(t)]=\lambda d t
\end{gathered}
$$

The invariance of the instantaneous mean of the transformed Poisson process under new measure $\bar{Q}$ is

$$
E_{\bar{Q}}[d \bar{N}(\bar{t})]=\lambda d \bar{t}
$$

using (21) and (22) equation (29) gives

$$
E_{Q}\left[\Gamma e^{\epsilon H}(N) d t+\Gamma^{*} e^{\epsilon H}(N) d N(t)\right]=\lambda\left(\Gamma e^{\epsilon H}(t) d t+\Gamma^{*} e^{\epsilon H}(t) d N(t)\right) .
$$

Now expending (30) using (27) gives

$$
\left(\Gamma e^{\epsilon H}(N)+\lambda \Gamma^{*} e^{\epsilon H}(N)-\lambda\left(\Gamma e^{\epsilon H}(t)\right) d t=\Gamma^{*} e^{\epsilon H}(t) d N(t) .\right.
$$

Next we apply the invariant form to instantaneous variance of the transformed Poisson process measure i.e.,

$$
E_{\bar{Q}}[d \bar{N}(\bar{t}) d \bar{N}(\bar{t})]=\lambda d \bar{t}
$$

which using (22) gives

$$
E_{\bar{Q}}\left[\Gamma^{*} e^{\epsilon H}(N) \Gamma^{*} e^{\epsilon H}(N) d N(t) d N(t)\right]=\lambda d \bar{t},
$$

finally from (21) and (33) give the following differential relation

$$
\Gamma^{*} e^{\epsilon H}(N) \Gamma^{*} e^{\epsilon H}(N) d t=\Gamma e^{\epsilon H}(t) d t+\Gamma^{*} e^{\epsilon H}(t) d N(t) .
$$


Comparing the jump and Riemann integrals from (34) we have the following relations

$$
\Gamma^{*} e^{\epsilon H}(t)=0
$$

and

$$
\Gamma^{*} e^{\epsilon H}(N) \Gamma^{*} e^{\epsilon H}(N)=\Gamma e^{\epsilon H}(t) .
$$

Using (35), (31) reduced to

$$
\Gamma e^{\epsilon H}(N)+\lambda \Gamma^{*} e^{\epsilon H}(N)=\lambda \Gamma e^{\epsilon H}(t) .
$$

Thus we have successfully derived the generalised random time change formula

$$
\bar{t}=\int^{t} \Gamma e^{\epsilon H}(s) d s
$$

with

$$
\Gamma e^{\epsilon H}(t)=\text { constant }=c_{1}
$$

using the probabilistic invariance property of the transformed time index differential, i.e.

$$
E_{\bar{Q}}[d \bar{t}(t, N)]=d \bar{t} .
$$

Therefore the generalised infinitesimal jump process is

$$
d \bar{N}(\bar{t})=\lambda\left(\Gamma e^{\epsilon H}(t)-\Gamma^{*} e^{\epsilon H}(N)\right) d t+\Gamma^{*} e^{\epsilon H}(N) d N(t)
$$

with

$$
\Gamma^{*} e^{\epsilon H}(N) \Gamma^{*} e^{\epsilon H}(N)=\lambda \Gamma e^{\epsilon H}(t)
$$

This is a generalised random time change formula for the Poisson process.

\subsection{Invariance Form of the Spatial Process}

To ensure the recovery of the finite transformations from the infinitesimal transformation, we need to transform $d \bar{X}(\bar{t})$ into

$$
d \bar{X}(\bar{t}, \bar{N})=\bar{f}(\bar{t}, \bar{X}(\bar{t}, \bar{N})) d \bar{t}+\bar{J}(\bar{t}, \bar{X}(\bar{t}, \bar{N})) d \bar{N}(\bar{t})
$$

where the transformed drift component using our generator (17) is

$$
\bar{f}(\bar{t}, \bar{X}(\bar{t}, \bar{N}))=e^{\epsilon H}(f)
$$

and the transformed Poisson amplitude is

$$
\bar{J}(\bar{t}, \bar{X}(\bar{t}, \bar{N}))=e^{\epsilon H}(J)
$$


Expanding the drift component (44) we have

$$
\bar{f}(\bar{t}, \bar{X}(\bar{t})) \overline{d t}=(f(t, X)+\epsilon(\Gamma H(t)+H) f(t, X)) d t .
$$

The following condition is necessary to ensure the recovery of the finite transformations from the infinitesimal transformation

$$
e^{\epsilon H(t)}(t, X)=\Gamma\left(e^{\epsilon H(t)}(t, X)\right) \text {. }
$$

Condition (47) is to ensure that the higher order terms depend on the first term associated with $O(\epsilon)$. All the ordered terms contribute in the construction of the finite transformations, the zeroth and the first order terms contribute towards the construction of the infinitesimal transformations. This also forces the instantaneous drift coefficient of the temporal infinitesimal to be a constant. i.e.

$$
\Gamma(\tau)=c_{1}
$$

Expanding the Poisson amplitude (45) gives

$$
\begin{aligned}
J(\bar{t}, \bar{X}(\bar{t}, \bar{N})) d \bar{N}(\bar{t}) & =J f(t, X) d N+\lambda \epsilon\left(J \Gamma H(N)-J \Gamma^{*} H(N)\right) d t \\
& +\epsilon\left(J \Gamma^{*} H(t)+H(J)\right) d N(t) .
\end{aligned}
$$

Definition 3. The infinitesimals transformation

$$
\bar{t}=e^{\epsilon H}(t), \quad \bar{x}=e^{\epsilon H}(x) \quad \text { and } \quad \bar{N}=e^{\epsilon H}(N)
$$

are called admitted Lie symmetry transformations of stochastic differential equations driven by Poisson processes

$$
d X(t, N)=f(t, X(t, N)) d t+J(t, X(t, N)) d N(t)
$$

if they leaves (51) and infinitesimal moments of the Poisson process i.e.,

$$
E_{Q}[d N(t, N)]=\lambda d t \quad E_{Q}[d N(t) d N(t)]=\lambda d t \quad \text { and } \quad E_{Q}[d t]=d t
$$

invariant, where $\lambda>0$ is the Poisson jump intensity or simply the jump rate. 


\section{Determining Equations}

This section is devoted to finding the determining equations of the admitted Lie symmetries of the stochastic differential equation driven by Poisson processes (1).

The intention is to transform given SDE driven by Poisson processes (1) into

$$
d \bar{X}(\bar{t}, \bar{N})=\bar{f}(\bar{t}, \bar{X}(\bar{t}, \bar{N})) d \bar{t}+\bar{J}(\bar{t}, \bar{X}(\bar{t}, \bar{N})) d \bar{N}(\bar{t}) .
$$

This can be achieved by substituting (46) and (49) into (53) which gives

$$
\begin{aligned}
d \bar{X}(\bar{t}, \bar{N}) & =d X(t)+\epsilon\left(f \Gamma H(t)+H(f)+\lambda J \Gamma H(t)-\lambda J \Gamma^{*} H(N)\right) d t \\
& +\left(J \Gamma^{*} H(N)+H(J)\right) d N(t) .
\end{aligned}
$$

Therefore, by comparing equation (54) and (23) we have the following determining equations

$$
(f \Gamma(\tau)+\lambda J(\Gamma(\tau)-\Gamma(\phi))+H(f)-\Gamma(\xi))(t, X(t, N))=0
$$

and

$$
\left(J \Gamma^{*}(\phi)+H(J)-\Gamma^{*}(\xi)\right)(t, X(t, N))=0
$$

Equations (36) and (37) give

$$
\Gamma^{*}(\phi)+\Gamma^{*}(\phi)=\Gamma(\tau)
$$

and

$$
\Gamma(\phi)+\lambda \Gamma^{*}(\phi)=\lambda \Gamma(\tau)
$$

From (57) we have

$$
\Gamma^{*}(\phi)=\frac{\Gamma(\tau)}{2}
$$

Equations (58) and (59) give

$$
\Gamma(\phi)=\lambda \frac{\Gamma(\tau)}{2}
$$

To show the relationship between (55) and the first prolongation of ordinary differential equations we proceed by using the definition of first prolongation of an infinitesimal generator for non-stochastic ordinary differential equations

$$
H^{[1]}=H+\eta^{[1]} \frac{\partial}{\partial \dot{x}}
$$


Where

$$
\dot{x}=\frac{d x}{d t}=D_{t} x
$$

and

$$
\begin{array}{r}
\eta^{[1]}=D_{t}(\xi)-\dot{x} D_{t}(\tau) \\
=\frac{\partial \xi}{\partial t}+\dot{x} \frac{\partial \xi}{\partial x}-\dot{x}\left(\frac{\partial \tau}{\partial t}+\dot{x} \frac{\partial \tau}{\partial x}\right)
\end{array}
$$

with total time derivative $D_{t}$ defined as

$$
D_{t}=\frac{\partial}{\partial t}+\dot{x} \frac{\partial}{\partial x}+\ddot{x} \frac{\partial}{\partial \dot{x}}+\ldots
$$

using the definition of first prolongation on $(\dot{x}-f)$ at $\dot{x}=f$, can be expressed as

$$
\left.H^{[1]}(\dot{x}-f)\right|_{\dot{x}=f}=\eta^{[1]}-H(f) .
$$

Using (66) and (63) equation (55) can be write as

$$
\left.H^{[1]}(\dot{x}-f)\right|_{\dot{x}=f}-\lambda J(\Gamma(\tau)-\Gamma(\phi))=0 .
$$

Where $\Gamma(t, X(t, N))$ and $\Gamma^{*}(t, X(t, N))$ are defined using the operators (5) and (6) respectively, and $\lambda>0$ is called the jump intensity.

\section{Applications}

\subsection{Example One}

Consider

$$
d X(t, N)=-k t^{2} d t+b d N(t)
$$

with $b \neq 0$ and initial condition $X(0)=x_{0}$.

Using the determining equations (55), (56), (59) and (60) we get respectively

$$
\begin{gathered}
-k t^{2}\left(\frac{\partial \tau}{\partial t}-k t^{2} \frac{\partial \tau}{\partial x}\right)+b \lambda\left(\frac{\partial \tau}{\partial t}-k t^{2} \frac{\partial \tau}{\partial x}-\left(\frac{\partial \phi}{\partial t}-k t^{2} \frac{\partial \phi}{\partial x}\right)\right)-2 k t \tau=\left(\frac{\partial \xi}{\partial t}-k t^{2} \frac{\partial \xi}{\partial x}\right) \\
b(\phi(t, x+b, N)-\phi(t, x, N))=\xi(t, x+b, N)-\xi(t, x, N) \\
\phi(t, x+b, N)-\phi(t, x, N)=\frac{1}{2}\left(\frac{\partial \tau}{\partial t}-k t^{2} \frac{\partial \tau}{\partial x}\right)
\end{gathered}
$$


and

$$
\left(\frac{\partial \phi}{\partial t}-k t^{2} \frac{\partial \phi}{\partial x}\right)=\frac{\lambda}{2}\left(\frac{\partial \tau}{\partial t}-k t^{2} \frac{\partial \tau}{\partial x}\right)
$$

From (35) we have

$$
\frac{\partial \tau(t, x, N)}{\partial x}=0
$$

therefore, using (73) and (48) we have the temporal infinitesimal as

$$
\tau(t, N)=c_{1} t+c_{0} F_{1}(N) .
$$

Using (74) equations (69), (71) and (72) can be rewritten as $c_{1}\left(b \lambda-3 k t^{2}\right)-b \lambda\left(\phi_{t}(t, x, N)-k t^{2} \phi_{x}(t, x, N)\right)-2 k t f(n)=\xi_{t}(t, x, N)-k t^{2} \xi_{x}(t, x, N)$,

$$
\phi_{t}(t, x, N)-k t^{2} \phi_{x}(t, x, N)=\frac{\lambda c_{1}}{2}
$$

and

$$
\phi(t, x+b, N)-\phi(t, x, n)=\frac{c_{1}}{2} .
$$

Differentiating (75) and (76) respectively with respect to $x$ gives

$$
\begin{gathered}
-b \lambda\left(\phi_{t x}(t, x, N)-k t^{2} \phi_{x x}(t, x, N)\right)=\xi_{t x}(t, x, N)-k t^{2} \xi_{x x}(t, x, N), \\
\phi_{t x}(t, x, N)-k t^{2} \phi_{x x}(t, x, N)=0 .
\end{gathered}
$$

Substituting (79) into (78) gives

$$
\xi_{t x}(t, x, N)-k t^{2} \xi_{x x}(t, x, N)=0 .
$$

Differentiating (77) with respect to $x$ gives

$$
\phi_{x}(t, x+b, N)=\phi_{x}(t, x, N)=g(t, N) .
$$

Differentiating (70) with respect to $x$ and using (68) gives

$$
\xi_{x}(t, x+b, N)=\xi_{x}(t, x, N)=h(t, N) .
$$

From (81) and (82) we have

$$
\phi_{x x}(t, x, N)=0 \quad \text { and } \quad \xi_{x x}(t, x, N)=0,
$$

which implies from (79) and (80)

$$
\phi_{x t}(t, x, N)=0 \quad \text { and } \quad \xi_{x t}(t, x, N)=0 .
$$


Therefore from (81) and (82) using (84) we get

$$
g(t, N)=g(N) \quad \text { and } \quad h(t, N)=h(N) .
$$

Which implies from (81) and (82) respectively

$$
\phi(t, x, N)=g(N) x+g_{1}(t, N),
$$

and

$$
\xi(t, x, N)=h(N) x+h_{1}(t, N) .
$$

Substituting (86) into (76) gives

$$
g_{1}(t, N)=\frac{k t^{3} g(N)}{3}+\frac{\lambda c_{1} t}{2}+g_{2}(N)
$$

therefore using (88) in (86) gives

$$
\phi(t, x, N)=g(N) x+\frac{k t^{3} g(N)}{3}+\frac{\lambda c_{1} t}{2}+g_{2}(N) .
$$

Substituting (89) into (77) gives the following relation

$$
g(N)=\frac{c_{1}}{2 b}
$$

Substituting (90) in (89) yields the infinitesimal of the Poisson process as

$$
\phi(t, x, N)=c_{1}\left(\frac{x}{2 b}+\frac{k t^{3}}{6 b}+\frac{\lambda t}{2}\right)+g_{2}(N) .
$$

Substituting (91) and (87) into (70) give the following relation

$$
h(N)=\frac{c_{1}}{2}
$$

Therefore from (87) using (92) yields

$$
\xi(t, x, N)=\frac{c_{1} x}{2}+h_{1}(t, N)
$$

Substituting (93) and (91) into (75) gives

$$
h_{1}(t, N)=c_{1}\left(\frac{-5 k t^{3}}{6}+\frac{b \lambda(2-\lambda) t}{2}\right)-k t^{2} f(N)+h_{2}(N) .
$$


Finally, from (93) using (94) yields the infinitesimal of the spatial variable as

$$
\xi(t, x, N)=c_{1}\left(\frac{x}{2}+\frac{-5 k t^{3}}{6}+\frac{b \lambda(2-\lambda) t}{2}\right)-k t^{2} f(N)+h_{2}(N) .
$$

So we have four symmetry generators as

$$
\begin{aligned}
& H_{1}=t \frac{\partial}{\partial t}+\left(\frac{x}{2}+\frac{-5 k t^{3}}{6}+\frac{b \lambda(2-\lambda) t}{2}\right) \frac{\partial}{\partial x}+\left(\frac{x}{2 b}+\frac{k t^{3}}{6 b}+\frac{\lambda t}{2}\right) \frac{\partial}{\partial N} \\
& H_{2}=f(N) \frac{\partial}{\partial t}-k t^{2} f(N) \frac{\partial}{\partial x} \quad H_{3}=h_{2}(N) \frac{\partial}{\partial x} \quad H_{4}=g_{2}(N) \frac{\partial}{\partial N} .
\end{aligned}
$$

\subsection{Example Two}

Consider a model

$$
d X(t, N)=-k^{2} t d t+\alpha t d N(t), \quad \alpha \neq 0
$$

with initial condition $X(0)=x_{0}$.

Using the determining equations (55), (56), (59) and (60) we get respectively

$$
\begin{gathered}
-k^{2} t\left(\frac{\partial \tau}{\partial t}-k^{2} t \frac{\partial \tau}{\partial x}\right)+\alpha \lambda t\left(\frac{\partial \tau}{\partial t}-k^{2} t \frac{\partial \tau}{\partial x}-\left(\frac{\partial \phi}{\partial t}-k^{2} t \frac{\partial \phi}{\partial x}\right)\right)-k^{2} \tau=\left(\frac{\partial \xi}{\partial t}-k^{2} t \frac{\partial \xi}{\partial x}\right) \\
\alpha t(\phi(t, x+\alpha t, N)-\phi(t, x, N))+\alpha \tau=\xi(t, x+\alpha t, N)-\xi(t, x, N) \\
\phi(t, x+\alpha t, N)-\phi(t, x, N)=\frac{1}{2}\left(\frac{\partial \tau}{\partial t}-k^{2} t \frac{\partial \tau}{\partial x}\right)
\end{gathered}
$$

and

$$
\left(\frac{\partial \phi}{\partial t}-k^{2} t \frac{\partial \phi}{\partial x}\right)=\frac{\lambda}{2}\left(\frac{\partial \tau}{\partial t}-k^{2} t \frac{\partial \tau}{\partial x}\right) .
$$

From (35) we have

$$
\frac{\partial \tau(t, x, N)}{\partial x}=0 .
$$

Therefore, using (103) and (48) the infinitesimal of the temporal variable becomes

$$
\tau(t, N)=c_{1} t+c_{0} F_{1}(N) .
$$

Using (104) equation (99), (101 and (102) can be rewritten as

$$
-k^{2} t c_{1}+\alpha \lambda t\left(c_{1}-\left(\frac{\partial \phi}{\partial t}-k^{2} t \frac{\partial \phi}{\partial x}\right)\right)-k^{2}\left(c_{1} t+c_{0} F_{1}(N)\right)=\left(\frac{\partial \xi}{\partial t}-k^{2} t \frac{\partial \xi}{\partial x}\right),
$$




$$
\begin{aligned}
\alpha t(\phi(t, x+\alpha t, N)-\phi(t, x, N))+\alpha\left(c_{1} t+c_{0} F_{1}(N)\right) & =\xi(t, x+\alpha t, N)-\xi(t, x, N), \\
\phi(t, x+\alpha t, N)-\phi(t, x, N) & =\frac{c_{1}}{2}
\end{aligned}
$$

and

$$
\left(\frac{\partial \phi}{\partial t}-k^{2} t \frac{\partial \phi}{\partial x}\right)=\frac{c_{1} \lambda}{2} .
$$

Differentiating (107) and (106) with respect to $x$ gives

$$
\left.\phi_{x}(t, x+\alpha t, N)=\phi_{x}(t, x, N)\right)=g(t, N)
$$

and

$$
\alpha t\left(\phi_{x}(t, x+\alpha t, N)-\phi_{x}(t, x, N)\right)=\xi_{x}(t, x+\alpha t, N)-\xi_{x}(t, x, N) .
$$

Using (109) in (110) yields

$$
\xi_{x}(t, x+\alpha t, N)=\xi_{x}(t, x, N)=h(t, N) .
$$

Similarly using (109) into (108) gives

$$
\phi_{t x}=0
$$

which implies

$$
\phi(t, x, N)=g_{1}(N) x+g_{2}(t, N) .
$$

Substituting (113) in (107) we get

$$
g_{1}(N)=c_{1}=0
$$

which implies

$$
\phi(t, x, N)=g_{2}(t, N) .
$$

Similarly substituting (115) into (108) gives

$$
\phi(t, x, N)=g_{3}(N) .
$$

solving (109) yields

$$
\xi(t, x, N)=h(t, N) x+h_{1}(t, N) .
$$

Substituting (117) in (106) using (114) and (116) gives

$$
h(t, N)=\frac{c_{0} f(N)}{t} .
$$


Therefore substituting (118) into (117) we have

$$
\xi(t, x, N)=\frac{c_{0} f(N)}{t} x+h_{1}(t, N)
$$

Substituting (118) into (105) using (114) and (116) gives

$$
c_{0}=0 \quad \text { and } \quad h_{1}(t, N)=h_{2}(N) .
$$

Using (114) and (120) the infinitesimals of the temporal and spatial variables (104) and (119) respectively become

$$
\tau(t, x, N)=0 \quad \text { and } \quad \xi(t, x, n)=h_{2}(N)
$$

therefore, we have two symmetry infinitesimal generators as

$$
H_{1}=h_{2}(N) \frac{\partial}{\partial x} \quad H_{2}=g_{3}(N) \frac{\partial}{\partial x} .
$$

\subsection{Example Three}

Consider

$$
d X(t, N)=-k^{2} x d t+\sqrt{2 k^{2}} d N(t), \quad k \neq 0
$$

where $k$ is a non-negative real number and initial condition $X(0)=x_{0}$.

Using the determining equations (55), (56), (59) and (60) we respectively yields

$$
\begin{gathered}
-k^{2} x\left(\frac{\partial \tau}{\partial t}-k^{2} x \frac{\partial \tau}{\partial x}\right)+\sqrt{2 k^{2}} \lambda_{0}\left(\frac{\partial \tau}{\partial t}-k^{2} x \frac{\partial \tau}{\partial x}-\left(\frac{\partial \phi}{\partial t}-k^{2} x \frac{\partial \phi}{\partial x}\right)\right)-k^{2} \xi=\left(\frac{\partial \xi}{\partial t}-k^{2} x \frac{\partial \xi}{\partial x}\right. \\
\sqrt{2 k^{2}}\left(\phi\left(t, x+\sqrt{2 k^{2}}, N\right)-\phi(t, n, x)\right)=\xi\left(t, x+\sqrt{2 k^{2}}, N\right)-\xi(t, x, N) \\
\phi\left(t, x+\sqrt{2 k^{2}}, N\right)-\phi(t, x, N)=\frac{1}{2}\left(\frac{\partial \tau}{\partial t}-k^{2} x \frac{\partial \tau}{\partial x}\right)
\end{gathered}
$$

and

$$
\left(\frac{\partial \phi}{\partial t}-k^{2} x \frac{\partial \phi}{\partial x}\right)=\frac{\lambda}{2}\left(\frac{\partial \tau}{\partial t}-k^{2} x \frac{\partial \tau}{\partial x}\right) .
$$

From (35) we have

$$
\frac{\partial \tau(t, x, N)}{\partial x}=0 .
$$

Therefore using (128) and (48) we have the temporal infinitesimal as

$$
\tau(t, N)=c_{1} t+c_{0} F_{1}(N) .
$$


Using (129) equation (124), (126) and (127) can be rewritten respectively as

$$
\begin{aligned}
-k^{2} x c_{1}+\sqrt{2 k^{2}} \lambda\left(c_{1}-\left(\frac{\partial \phi}{\partial t}-k^{2} x \frac{\partial \phi}{\partial x}\right)\right)-k^{2} \xi & =\left(\frac{\partial \xi}{\partial t}-k^{2} x \frac{\partial \xi}{\partial x}\right) \\
\phi\left(t, x+\sqrt{2 k^{2}}, N\right)-\phi(t, x, N) & =\frac{c_{1}}{2}
\end{aligned}
$$

and

$$
\left(\frac{\partial \phi}{\partial t}-k^{2} x \frac{\partial \phi}{\partial x}\right)=\frac{c_{1} \lambda}{2} .
$$

Differentiating (125) and (131) with respect to $x$ respectively gives

$$
\sqrt{2 k^{2}}\left(\phi_{x}\left(t, x+\sqrt{2 k^{2}}, N\right)-\phi_{x}(t, x, N)\right)=\xi_{x}\left(t, x+\sqrt{2 k^{2}}, N\right)-\xi_{x}(t, x, N)
$$

and

$$
\phi_{x}\left(t, x+\sqrt{2 k^{2}}, x\right)=\phi_{x}(t, x, N)=g(t, N) .
$$

Substituting (134) into (133) we get

$$
\xi_{x}\left(t, x+\sqrt{2 k^{2}}, x\right)=\xi_{x}(t, x, N)=h(t, N) .
$$

Differentiating (130) and (132) with respect to $x$ respectively gives

$$
-k^{2} c_{1}-\left(\phi_{t x}-x k^{2} \phi_{x x}-k^{2} \phi_{x}\right)=\xi_{t x}-x k^{2} \xi_{x} x,
$$

and

$$
\phi_{t x}-x k^{2} \phi_{x x}-k^{2} \phi_{x}=0 .
$$

Substituting (137) into (136) gives

$$
\xi_{t x}-x k^{2} \xi_{x x}+k^{2} c_{1}=0 .
$$

From (134) and (135) we have

$$
\phi_{x x}=0 \quad \text { and } \quad \xi_{x x}=0 .
$$

Substituting (139) in (137) and (138) respectively yields

$$
\phi_{t x}-k^{2} \phi_{x}=0
$$

and

$$
\xi_{t x}+k^{2} c_{1}=0
$$


Solving (140) and (141) respectively using (134) and (135) yields

$$
\phi=g_{1}(N) e^{k^{2} t} x+g_{2}(t, N)
$$

and

$$
\xi(t, x, N)=-k^{2} x t c_{1}+x h_{1}(n)+h_{2}(t, n) .
$$

Substituting (142) in (132) gives

$$
g_{2}(t,(t, N))=\frac{c_{1} t \lambda}{2}+g_{3}(N) .
$$

Similarly substituting (142) in (131) using (144) gives

$$
g_{1}(N)=c_{1}=0
$$

therefore, using (145) and (142) the infinitesimal of the Poisson process reduced to

$$
\phi=g_{3}(N)
$$

Using (146), equation (133) implies

$$
\xi\left(t, x+\sqrt{2 k^{2}}, x\right)-\xi(t, x, N)=0 .
$$

Substituting into (143) from (147) yields

$$
h_{1}(N)=0
$$

Therefore using (145) and (148) gives

$$
\xi=h_{2}(t, N)
$$

Finally, substituting (149) into (130) using (145) and (146) yields the spatial infinitesimal as

$$
\xi(t, N)=h_{2}(t, N)=h_{3}(N) e^{-k^{2} t}
$$

So we have three symmetry infinitesimal generators as

$$
H_{1}=f(N) \frac{\partial}{\partial t}, \quad H_{2}=h_{3}(N) e^{-k^{2} t} \frac{\partial}{\partial x}, \quad H_{3}=g_{3}(N) \frac{\partial}{\partial N}
$$




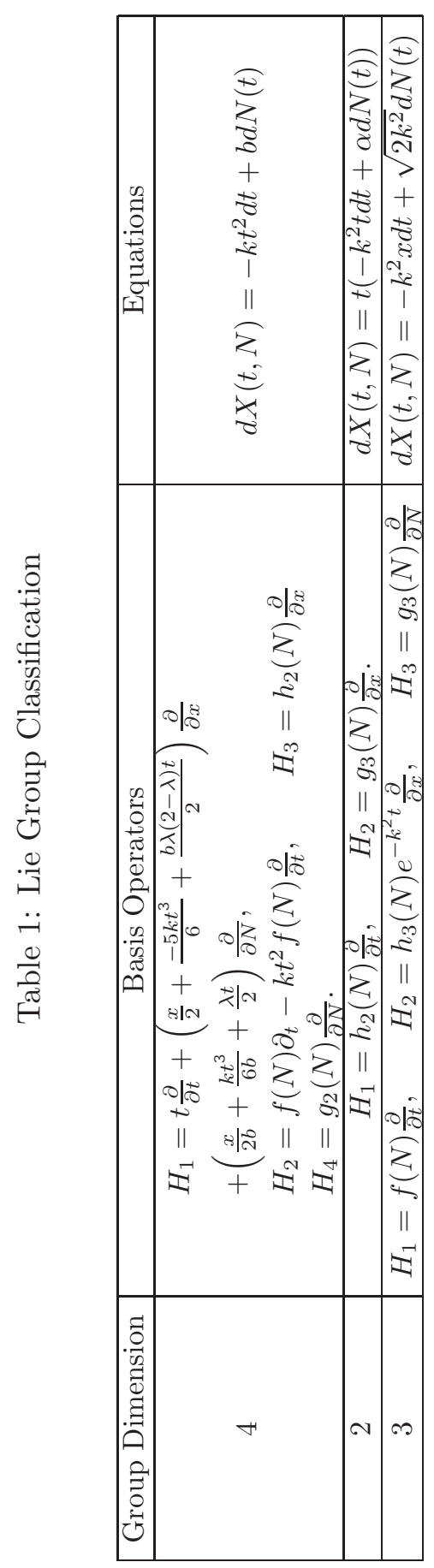




\section{Conclusion}

Lie point symmetry transformations for the class of Itô stochastic differential equations driven by Poisson Processes was successfully carried out. We considered symmetries involving not only spatial $x$ and time variables $t$, but also included the Poisson process term $N(t)$. This was achieved by following the methodology of Lie point transformations [3, 8] and the use of Itô formula for Poisson stochastic differential equations [1] without enforcing any conditions at the momenta of the stochastic processes.

We ensured the instantaneous mean and variance of the Poisson stochastic processes remained invariant under the transformation (16).

Finally, the determining equations for the Itô stochastic differential equation driven by Poisson processes

$$
d X_{i}(t, N)=f_{i}(t, X(t, N)) d t+J_{i}(t, X(t, N)) d N(t)
$$

were successfully derived and they were found to be non-stochastic even though they represent stochastic processes. Determining equations found were later applied to a few examples which, while simple, are non-trivial to find their correspondent admitted Lie symmetries. Classification of the given examples are presented in Table 1 below.

\section{References}

[1] B. Hanson Floyd, Applied Stochastic Processes and Control for Jump-Diffusions: Modeling, Analysis and Computation, Society for Industrial and Applied Mathematics, (2007).

[2] P. E. Kloeden, E. Platen, Numerical Solution of Stochastic Differential Equations Springer, Applications of Mathematics 64, (2010).

[3] E. Fredericks, F. M. Mahomed, A Formal Approach for Handling Lie Point Symmetries of Scalar First-Order Itô Stochastic Ordinary Differential Equations, Journal of Nonlinear Mathematical Physics Volume 15, (2008), 44-59.

[4] G. Gaeta, Symmetry of Stochastic Equations, Journal of Proceedings National Academy of Sciences Ukraine, 50, (2004), 98-109.

[5] M. Aminu Nass, E. Fredericks : Lie Symmetry of Itô Stochastic Differential Equation Driven by Poisson Process, American Review of Mathematics and Statistics, 4(1) (2016).

[6] G. Gaeta, QR. Quintero, Lie-point Symmetries and Stochastic Differential Equations. J Phys A: Math Gen; 32, (1999), 485505.

[7] G. Ünal, A. Sanver, I. Iyigunler, Exact Linearization of One-dimensional Jump-diffusion Stochastic Different Equations, Non-linear Dynamics, 51(1-2), (2008), 1-8.

[8] Roman Kozlov, On Lie Group Classification of a Scalar Stochastic Differential Equation, Journal of Nonlinear Mathematical Physics, 18(sup1), (2011), 177-187. 